\title{
Radiation recall dermatitis occurring 6 years and 4 months after breast-conserving surgery: A case report
}

\author{
MAMIKO UBUKATA, TAKAKO KAMIO, TETSUYA OHCHI, EIICHIRO NOGUCHI, \\ HIROKO TSUKADA and SHINGO KAMEOKA
}

Department of Surgery II, Tokyo Women's Medical University, Tokyo 162-8666, Japan

Received March 9, 2015; Accepted November 23, 2015

DOI: $10.3892 / \mathrm{ol} .2016 .4346$

\begin{abstract}
Currently in Japan, breast-conserving therapy, consisting of breast-conserving surgery and post-operative radiation therapy, is performed frequently for the treatment of invasive breast cancer. It has been demonstrated that radiation therapy not only prevents recurrence in the preserved breast, but that it also contributes to improved patient survival. The present study describes the case of a 37-year-old woman with radiation recall dermatitis that occurred 6 years and 4 months after breast-conserving surgery. Erythema with a relatively distinct border was observed at the irradiated site on the left breast; eczema was diagnosed by a dermatologist. Inflammatory breast cancer was ruled out, since chest X-ray, abdominal ultrasound and bone scintigraphy were negative. Following $\sim 1$ month of topical corticosteroid application and oral second generation antihistamine treatment, the erythema was alleviated and the subjective symptoms also disappeared. Only a few cases of radiation recall dermatitis have been described in the fields of radiology and dermatology, but not yet in the surgical field. In the future, the incidence of radiation recall dermatitis is predicted to increase due to the increasing number of patients undergoing breast-conserving therapy. Whether in the surgical, radiological or dermatological field, if erythema is detected at the irradiated site during post-operative follow-up, routine care should be provided, keeping in mind the possibility of radiation recall dermatitis and inflammatory breast cancer.
\end{abstract}

Correspondence to: Professor Takako Kamio, Department of Surgery II, Tokyo Women's Medical University, 8-1 Kawada-cho, Shinjuku-ku, Tokyo 162-8666, Japan

E-mail: ako@surg2.twmu.ac.jp

Abbreviations: TAM, tamoxifen

Key words: breast cancer, radiation recall dermatitis, tamoxifen, breast-conserving surgery, tamoxifen-induced radiation recall dermatitis

\section{Introduction}

Since 2003, breast-conserving therapy (the combination of breast-conserving surgery and post-operative radiation therapy to the conserved breast) has been performed more frequently than mastectomy for the treatment of stage I or II invasive breast cancer (primarily tumors $\leq 3 \mathrm{~cm}$ ) in Japan (1). Furthermore, it has been observed that the combined usage of post-operative radiation not only prevents recurrence within the preserved breast, but that it also contributes to improved patient survival (1-3). Adverse events, including radiation dermatitis and secondary cancer, have been reported following the application of breast-conserving therapy; however, the majority of radiation dermatitis cases develop during the acute stages of the treatment and the mechanism of late occurrence is unknown (1). Radiation recall dermatitis has also been reported, which is a condition triggered by drug administration following radiation therapy, and is characterized by an inflammatory response that is localized to the irradiated site of the body (4-9).

The present study describes a case of radiation recall dermatitis that occurred 6 years and 4 months after breast-conserving surgery, along with a review of the relevant literature. Written informed consent was obtained for publication of the present study.

\section{Case report}

A 37-year-old woman was referred to the Department of Surgery II, Tokyo Women's Medical University (Tokyo, Japan) in 2014, presenting with scaly erythema and itching of the skin on the left breast (Fig. 1). The patient had undergone left breast-conserving surgery 6 years and 4 months previously for the treatment of breast cancer. It was noted that a relatively distinct border surrounded the erythema and was observed at the irradiated site on the left breast (Fig. 1).

In 2007, the patient underwent left breast-conserving surgery to treat breast cancer of the left upper outer quadrant, with a sentinel lymph node biopsy removed for analysis. The pathology showed invasive ductal carcinoma, T1b (7x6 mm); gland, lymphatic invasion, 0 ; venous invasion, 0 ; histological grade, $2+1+1$; lymph node, 0/1; estrogen receptor-positive, progesterone receptor-positive, human epidermal growth factor receptor 2-negative. Post-operatively, the patient was 


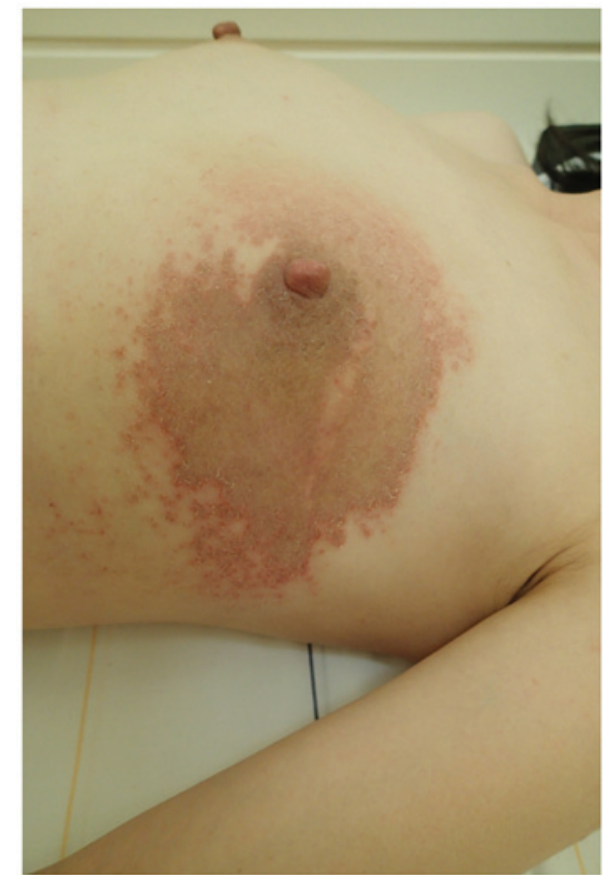

Figure 1. Radiation recall dermatitis at the initial visit. Scaly erythema, with a relatively distinct border, was observed at the irradiated site on the left breast.

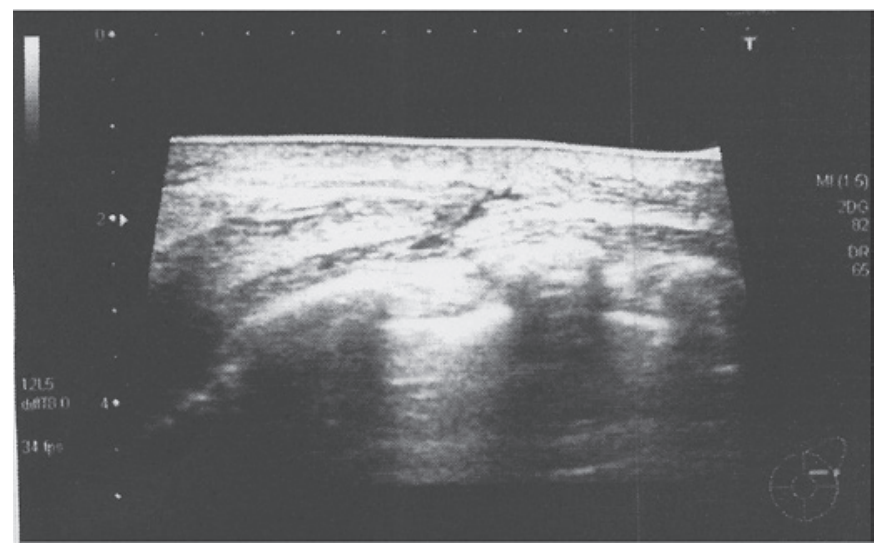

Figure 2. Breast ultrasonography. Skin hypertrophy was detected in the area of redness. No marked lesions were observed in the mammary gland.

treated with oral anti-estrogen (Nolvadex tablets, $20 \mathrm{mg} /$ day) for 5 years and a subcutaneous luteinizing hormone-releasing hormone agonist (Leuplin SR $11.25 \mathrm{mg}$ Injection kit) for 2 years. In addition, whole breast radiation + additional radiation [4 MV X-ray at 46 Gy (23 times) + 9 Gy (3 times)] was applied to the remaining breast tissue. Subsequently, follow-up was conducted on an outpatient basis. There was no history of pregnancy and also no significant family history noted.

During the present case, blood biochemistry demonstrated that the white blood cell count was 5,300/ $\mu 1$ (normal range, 4,000-8,600/ $\mu 1$ ), indicating that there was no inflammatory response, and all other measurements were within normal ranges. Furthermore, the carcinoembryonic antigen level was $0.8 \mathrm{ng} / \mathrm{ml}$ (normal range, $\leq 5.0 \mathrm{ng} / \mathrm{ml}$ ), and the cancer antigen $15-3$ level was $6.5 \mathrm{U} / \mathrm{ml}$ (normal range, $\leq 25.0 \mathrm{U} / \mathrm{ml}$ ), indicating that tumor markers were not elevated. Ultrasonography

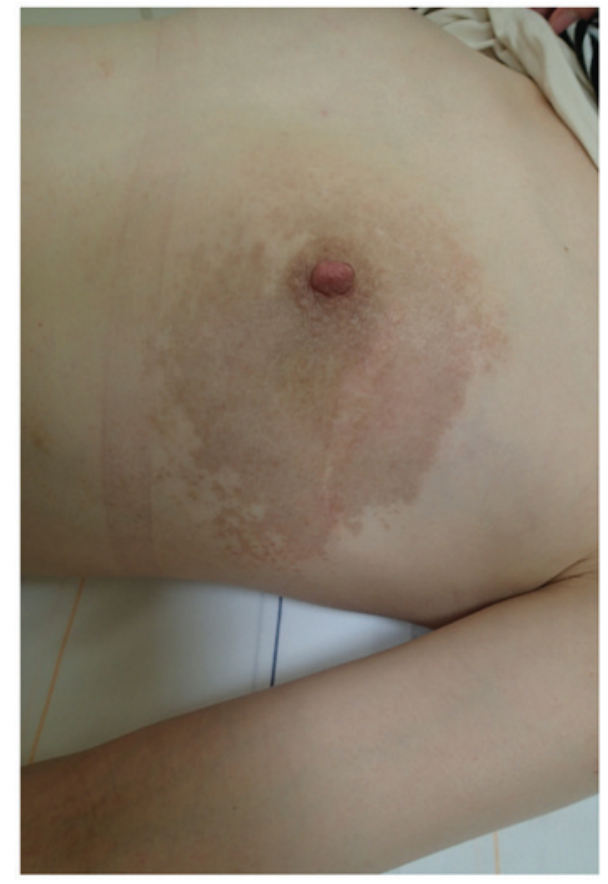

Figure 3. Skin of the affected breast subsequent to treatment. Following $\sim 1$ month of topical corticosteroid application + oral second generation antihistamine administration, the erythema was alleviated and the subjective symptoms (including itching) also disappeared.

of the breast detected skin hypertrophy in the area of redness (Fig. 2). No marked lesions were observed in the mammary gland.

Despite erythema on the skin of the affected breast initially suggesting the presence of inflammatory breast cancer, the patient did not exhibit any signs of inflammation, including burning sensations or pain. Breast ultrasonography only detected skin hypertrophy. Since there was no dilation observed in the lymphatic vessels, it was unlikely that the patient had inflammatory breast cancer. Furthermore, a full body examination was performed, including a chest X-ray, abdominal ultrasound and bone scintigraphy, confirming that there was no breast cancer recurrence.

Upon consultation with a dermatologist regarding the development of the rash, the patient was diagnosed with eczema. Following $\sim 1$ month of topical corticosteroid application (Antebate ointment $0.05 \%)+$ oral second-generation antihistamine treatment (Talion tablets, $10 \mathrm{mg} / \mathrm{day}$ ), the erythema was alleviated and the subjective symptoms, including the itching, also disappeared (Fig. 3). Written informed consent was obtained from the patient.

\section{Discussion}

Currently in Japan, breast-conserving therapy (breast-conserving surgery and post-operative radiation therapy to the conserved breast) is performed frequently for the treatment of invasive breast cancer (primarily tumors $\leq 3 \mathrm{~cm}$ in size). Furthermore, randomized, controlled studies have observed significant decreases in cancer recurrence in the preserved breast in all groups that received a combination of radiation therapy, and it has been concluded, domestically and internationally, that radiation treatments are necessary following breast-conserving surgery $(1,10)$. Additionally, it has 
been demonstrated that radiation therapy not only prevents recurrence in the preserved breast, but that it also contributes to improved patient survival (1-3).

Radiation dermatitis is categorized into two groups: Acute dermatitis and chronic dermatitis. Acute dermatitis develops at sites that have received large doses of radiation over a short period of time, whilst chronic dermatitis occurs at sites that have repeatedly received small doses of radiation over a long period of time. Radiation dermatitis and secondary cancer have been reported as adverse events of combined treatment with post-operative radiation, although the majority of radiation dermatitis cases are considered to appear at the acute stage of treatment (1).

To the best of our knowledge, there are no previous reports similar to the present case, where erythema with distinct borders has rapidly appeared at the irradiated area 6 years and 4 months after breast-conserving surgery ( 6 years and 2 months after radiation).

In the literature, a phenomenon called radiation recall dermatitis has been reported; this is a condition triggered by drug administration following radiation therapy, and is characterized by an inflammatory response localized to the irradiated body site (4-9). Although the precise mechanism underlying radiation recall dermatitis is unknown, it has been proposed that stem cell depletion, increased local vascular permeability, overexpression of transforming growth factor $\beta 1$ (TGF- $\beta 1$ ) or the expression of inflammatory cytokines may cause this condition $(4,5)$. It has also been hypothesized that the condition is triggered by specific drugs that are administered days to years after exposure to ionizing radiation (6).

In general, it is considered to be difficult to distinguish between a radiation-induced skin disorder and radiation recall dermatitis. Nonetheless, in clinical practice, the two conditions are often differentiated by focusing on the specific clinical course of radiation recall dermatitis and on the fact that the rash appears following the administration of certain drugs.

Drugs that initiate radiation recall dermatitis include anticancer drugs (taxanes), antibiotics and antituberculosis agents, with anticancer drugs accounting for $20-30 \%$ of cases (7).

In Western countries, four cases of tamoxifen (TAM)-induced radiation recall dermatitis have been reported in which the condition was triggered by the administration of antiestrogen following radiation therapy for breast cancer (Table I) $(4,5,8,9)$. There have been no reports thus far of any TAM-induced radiation recall dermatitis cases located in Japan.

In all four of the aforementioned cases, erythema appeared consistently at the site of irradiation within 3 months of initiating oral TAM therapy, which was administered either pre-operatively or post-operatively, and the rash promptly disappeared with TAM discontinuation, or with the administration of topical corticosteroids, oral antihistamines or oral antibiotics $(4,5,8,9)$.

Following careful examination of the rashes in the studies regarding radiation recall dermatitis (4-9), it is evident that, as in the present case, erythema with an itching sensation localized to the area of irradiation was observed in all cases. In addition, the patient in the present case had taken TAM over a 5 -year period post-operatively, and based on the evidence 
that the rash appeared following the initiation of post-radiation drug treatment, radiation recall dermatitis was suspected.

However, the patient in the present case had already completed the oral TAM treatment, and therefore the timing of oral TAM duration and rash development did not coincide, as observed in the four cases that have been reported as TAM-induced radiation recall dermatitis.

It has been suggested that a certain number of days after the completion of radiation treatment, the irradiated site becomes 'prepared' for radiation recall dermatitis, indicating that the late development of the rash may depend on external stimuli or the immune status of the patient, even in patients similar to the present case where a year had passed since the completion of oral medication (11).

In the present case, a skin biopsy was not performed. Since there are few reports regarding biopsies performed in cases of radiation recall dermatitis, information concerning the typical pathological findings was not located. However, if a skin biopsy had been performed, this would have permitted greater examination of the pathology, possibly allowing confirmation of the primary locus of inflammation in the skin tissue.

Radiation recall dermatitis has been described in a limited number of cases in the radiological and dermatological fields, but not in the surgical field. The incidence of this disorder is predicted to increase, primarily due to the increasing number of patients undergoing breast-conserving therapy, and also due to the extended indications for various drugs (including taxanes).

In conclusion, the present study reported a case of radiation recall dermatitis that developed 6 years and 4 months after breast-conserving surgery. It is predicted that the incidence of this disease will increase in the future. Therefore, when erythema appears during post-operative follow-up at an area that coincides with the irradiated site, routine medical care should be provided, keeping in mind the possibility of radiation recall dermatitis, in addition to inflammatory breast cancer.

\section{Acknowledgements}

The authors would like to gratefully acknowledge the assistance of Forte Science Communications (Tokyo, Japan) in the preparation of this manuscript.

\section{References}

1. The Japanese Breast Cancer Society: Clinical Practice Guidelines for Breast Cancer Treatment. 2nd edition. Kanehara \& Co., Ltd., 2013.

2. Vinh-Hung V and Verschraegen C: Breast-conserving surgery with or without radiotherapy: Pooled-analysis for risks of ipsilateral breast tumor recurrence and mortality. J Natl Cancer Inst 96: 115-121, 2004.

3. Early Breast Cancer Trialists' Collaborative Group (EBCTCG); Darby S, McGale P, Correa C, Taylor C, Arriagada R, Clarke M, Cutter D, Davies C, Ewertz M, Godwin J, et al: Effect of radiotherapy after breast-conserving surgery on 10-year recurrence and 15-year breast cancer death: Meta-analysis of individual patient data for 10,801 women in 17 randomised trials. Lancet 378: 1707-1716, 2011.

4. Kundranda MN and Daw HA: Tamoxifen-induced radiation recall dermatitis. Am J Clin Oncol 29: 637-638, 2006.

5. Singer EA, Warren RD, Pennanen MF, Collins BT and Hayes DF: Tamoxifen-induced radiation recall dermatitis. Breast J 10: 170-171, 2004.

6. Camidge $\mathrm{R}$ and Price A: Characterizing the phenomenon of radiation recall dermatitis. Radiother Oncol 59: 237-245, 2001.

7. Hird AE, Wilson J, Symons S, Sinclair E, Davis M and Chow E: Radiation recall dermatitis: Case report and review of the literature. Curr Oncol 15: 53-62, 2008.

8. Parry BR: Radiation recall induced by tamoxifen. Lancet 340 : 49, 1992.

9. Boström A, Sjölin-Forsberg G, Wilking N and Bergh J: Radiation recall - another call with tamoxifen. Acta Oncol 38: 955-959, 1999.

10. Clarke M, Collins R, Darby S, Davies C, Elphinstone P, Evans V, Godwin J, Gray R, Hicks C, James S, et al; Early Breast Cancer Trialists' Collaborative Group (EBCTCG): Effects of radiotherapy and of differences in the extent of surgery for early breast cancer on local recurrence and 15-year survival: An overview of the randomised trials. Lancet 366: 2087-2106, 2005.

11. Chihiro N, Ken I, Kazuto H and Goichi T: Radiation recall dermatitis induced by cetuximab in a patient with advanced colon carcinoma. Japanese J Clin Dermatol 66: 14-18, 2012 (In Japanese). 